\title{
Fast and stable manipulation of a charged particle in a Penning trap
}

\author{
A. Kiely, ${ }^{1, \text { I J. P. L. McGuinness, }}{ }^{2}$ J. G. Muga, ${ }^{3,4}$ and A. Ruschhaupt ${ }^{1}$ \\ ${ }^{1}$ Department of Physics, University College Cork, Cork, Ireland \\ ${ }^{2}$ School of Mathematical Sciences, University College Cork, Cork, Ireland \\ ${ }^{3}$ Departamento de Química Física, UPV/EHU, Apdo 644, 48080 Bilbao, Spain \\ ${ }^{4}$ Department of Physics, Shanghai University, 200444 Shanghai, People's Republic of China
}

\begin{abstract}
We propose shortcuts to adiabaticity which achieve fast and stable control of the state of a charged particle in an electromagnetic field. In particular we design a non-adiabatic change of the magnetic field strength in a Penning trap which changes the radial spread without final excitations. We apply a streamlined version of the fast-forward formalism as well as an invariant based inverse engineering approach. We compare both methods and examine their stability.

PACS numbers: 42.50.Dv, 37.10.Ty, 37.10.Vz
\end{abstract}

\section{INTRODUCTION}

The coherent manipulation of quantum systems for quantum technologies, fundamental studies, or metrology often requires control protocols of external parameters that are fast and stable with respect to perturbations. This has motivated the development of shortcuts to adiabaticity (STA), which are schemes that reach the fidelities of adiabatic processes in shorter times, keeping or even enhancing their stability; shortcuts to adiabaticity have been reviewed in [1].

The fast expansion or compression of a particle state driven by a time dependent trap frequency is one of the paradigmatic operations for which STA have been developed, both in theory [2] and experiment [3, 4]. Interacting particles in an expanding/compressing external harmonic potential such as ion chains [5], Bose-Einstein condensates [6], or classical gases [7], have also been studied. There are many applications for fast expansion/compression, such as controlled cooling/heating of the state [8, 9] , implementation of quantum engines and refrigerators based on cyclic expansions and compressions [10, 11], fast switching between manipulations suited for a low trap frequency or for a high trap frequency configuration [5], or efficient sympathetic cooling [12, 13]. Fundamental aspects such as the quantification of the third law of thermodynamics [10, 14] have also been examined.

Real traps are of course three-dimensional, but most of the theory work deals with 1D traps with time dependent frequencies whose effective realization is not straightforward. Torrontegui et al. [15] studied the fast expansions of cold atoms in a three-dimensional Gaussian-beam optical trap. The radial and axial frequencies are coupled and as a consequence some shortcut schemes that work in 1D were in fact restricted to certain parameter domains, and others failed completely. Traps with uncoupled radial and axial frequencies are of interest to perform clean STA expansions/compressions and such a possibility is

*Electronic address: anthony.kiely@umail.ucc.ie indeed provided by the Penning trap.

In this paper, we will put forward shortcut schemes to control a charged particle in a Penning trap. A Penning trap uses a combination of a uniform and unidirectional magnetic field and an electrostatic quadrupole potential. This potential is typically created using three electrodes which are hyperboloids of revolution. Penning traps are commonly used for accurate measurement of the properties of different charged particles [16].

Since different operations on the trapped charged particle (preparation, measurement, or interactions) may require or benefit from different extensions of the density cloud, our aim is to change this extension rapidly without producing final excitations. Therefore, we shall construct schemes to decrease the radial extension of the particle's wave function, without producing final excitations, by changing the magnetic field intensity.

We shall first design such shortcuts by means of the fast-forward formalism. The basic fast-forward formalism for a particle in a time dependent potential (without an electromagnetic field) was first introduced by S. Masuda and K. Nakamura [17, 18]. Later a streamlined version of this formalism was developed in [19]. This streamlined formalism has been applied for example to engineering of fast and stable splitting of matter waves 20] and to achieve rapid loading of a Bose-Einstein condensate into an optical lattice [21]. Another strategy to design shortcut schemes is based on Lewis-Riesenfeld invariants [2, 22]. Applications of this strategy are reviewed in [1].

In the following section we will develop a streamlined version of the fast-forward formalism in the general case of a charged particle in an electromagnetic field. In Section III, we will review the known eigenstates of a particle in a a Penning trap. In section IV, we apply the streamlined formalism to change the state from an eigenstate of one magnetic field strength to that of a larger field strength. We also compare it with an inverse engineering approach based on Lewis-Riesenfeld invariants and examine the stability versus systematic errors in the magnetic field. Finally, in Section $\nabla \nabla$ we discuss our results. 


\section{GENERAL STREAMLINED FORMALISM}

A fast-forward formalism including an electromagnetic field was introduced in [23]. We shall now put forward a streamlined version.

\section{A. Main equations}

We consider a spinless charged particle as spin will not play any role in the trap configuration considered below. The Schrödinger equation for this particle in an electromagnetic field is given by

$$
i \hbar \frac{\partial}{\partial t} \Psi=H(t) \Psi
$$

where the Hamiltonian (expressed in some chosen gauge e.g. the Coulomb gauge) is given in coordinate representation by

$$
H(t)=\frac{1}{2 m}\left(\frac{\hbar}{i} \nabla-q \vec{A}(t, \vec{r})\right)^{2}+q \phi(t, \vec{r}),
$$

with $q$ being the charge, $\vec{A}$ the vector potential and $\phi$ the scalar potential (both real). We write $\Psi$ as

$$
\Psi(t, \vec{r})=\alpha(t, \vec{r}) e^{i \beta(t, \vec{r})}
$$

where $\alpha(t, \vec{r}), \beta(t, \vec{r}) \in \mathbb{R}$. Note that $\Psi(t, \vec{r})$ corresponds to the fast-forwarded state $\Psi_{F F}$ in [23]. $\vec{A}$ and $q \phi$ correspond to the driving potentials $\vec{A}_{F F}$ and $V_{F F}$ in [23].

Inserting the ansatz (3) into Eq. (11) and then multiplying the equation by $e^{-i \beta(t, \vec{r})}$, we get for the real part of the result

$0=-\frac{\hbar^{2}}{2 m} \Delta \alpha+\frac{1}{2 m}(q \vec{A}-\hbar \nabla \beta)^{2} \alpha+\left(q \phi+\hbar \frac{\partial \beta}{\partial t}\right) \alpha$

and for the imaginary part

$$
\hbar \frac{\partial \alpha}{\partial t}=\frac{\hbar}{2 m} \nabla(q \vec{A}-\hbar \nabla \beta) \alpha+\frac{\hbar}{m}(q \vec{A}-\hbar \nabla \beta) \nabla \alpha .
$$

To write these two equations in a more compact way, let us define

$$
\vec{\chi}:=\vec{A}-\frac{\hbar}{q} \nabla \beta, \Phi:=\phi+\frac{\hbar}{q} \frac{\partial \beta}{\partial t} .
$$

The electric and the magnetic fields are now given by

$$
\vec{E}=-\nabla \Phi-\frac{\partial \vec{\chi}}{\partial t}, \vec{B}=\nabla \times \vec{\chi}
$$

Using these definitions of $\vec{\chi}$ and $\Phi$, the two equations (4) and (5) simplify to

$$
\Phi=\frac{\hbar^{2}}{2 m q \alpha} \Delta \alpha-\frac{q}{2 m} \vec{\chi}^{2}
$$

and

$$
\frac{\partial \alpha}{\partial t}-\frac{q}{2 m}(\nabla \vec{\chi}) \alpha-\frac{q}{m} \vec{\chi} \nabla \alpha=0
$$

These are the two main equations.

Note that $\vec{\chi}$ and $\Phi$ as well as the main equations (8) and (9) are invariant under a gauge transformation $\Lambda$ acting in the usual way

$$
\vec{A} \rightarrow \vec{A}+\nabla \Lambda, \phi \rightarrow \phi-\frac{\partial \Lambda}{\partial t} \text { and } \Psi \rightarrow e^{\frac{i}{\hbar} q \Lambda} \Psi
$$

i.e., $\beta \rightarrow \beta+\frac{q}{\hbar} \Lambda$ and $\alpha$ is unchanged.

\section{B. Inverse engineering and boundary conditions}

Let the initial state of the system $\psi_{0}(\vec{r}) \equiv \alpha_{0}(\vec{r}) e^{i \beta_{0}(\vec{r})}$ be an eigenstate of the initial time independent Hamiltonian

$$
H_{0}=\frac{1}{2 m}\left(\frac{\hbar}{i} \nabla-q \overrightarrow{A_{0}}\right)^{2}+q \phi_{0},
$$

with eigenvalue $\mathcal{E}_{0}$. (The eigenstates of the Penning trap are reviewed in the following section.) The goal is to design a scheme $(\vec{A}(t, \vec{r})$ and $\phi(t, \vec{r}))$ such that the final state $($ at $t=T)$ of the system, $\psi_{T}(\vec{r}) \equiv \alpha_{T}(\vec{r}) e^{i \beta_{T}(\vec{r})}$, is an eigenstate of the final (time independent) Hamiltonian

$$
H_{T}=\frac{1}{2 m}\left(\frac{\hbar}{i} \nabla-q \vec{A}_{T}\right)^{2}+q \phi_{T}
$$

with eigenvalue $\mathcal{E}_{T}$. The Hamiltonian should be continuous at initial and final time, i.e., $H(0)=H_{0}$ and $H(T)=H_{T}$.

In the inversion protocol we first design $\alpha(t, \vec{r})$ and $\beta(t, \vec{r})$ fulfilling the boundary conditions

$$
\begin{gathered}
\alpha(0, \vec{r})=\alpha_{0}(\vec{r}), \alpha(T, \vec{r})=\alpha_{T}(\vec{r}), \\
\beta(0, \vec{r})=\beta_{0}(\vec{r}), \beta(T, \vec{r})=\beta_{T}(\vec{r}) .
\end{gathered}
$$

In the next step, we have to solve for $\vec{\chi}$ in Eq. (9). The function $\Phi$ is then given by Eq. (8). Because the Hamiltonian should be changing continuously at initial and final time, $\vec{\chi}$ must fulfill the following boundary conditions

$$
\vec{\chi}(0, \vec{r})=\vec{A}_{0}-\frac{\hbar}{q} \nabla \beta_{0}, \vec{\chi}(T, \vec{r})=\vec{A}_{T}-\frac{\hbar}{q} \nabla \beta_{T} .
$$

A consequence of these conditions can be seen by evaluating Eq.(9) at the initial and final time (see also Appendix A). This leads to

$$
\frac{\partial \alpha}{\partial t}(0, \vec{r})=0, \frac{\partial \alpha}{\partial t}(T, \vec{r})=0 .
$$

The boundary conditions of $\Phi$ can be seen by evaluating Eq. (8) at initial and final time leading to (see also 
Appendix (A)

$$
\begin{gathered}
\Phi(0, \vec{r})=\phi_{0}(\vec{r})-\frac{1}{q} \mathcal{E}_{0}=\phi_{0}(\vec{r})+\frac{\hbar}{q} \frac{\partial \beta}{\partial t}(0, \vec{r}), \\
\Phi(T, \vec{r})=\phi_{0}(\vec{r})-\frac{1}{q} \mathcal{E}_{T}=\phi_{0}(\vec{r})+\frac{\hbar}{q} \frac{\partial \beta}{\partial t}(T, \vec{r}) .
\end{gathered}
$$

These conditions are equivalent to

$$
\frac{\partial \beta}{\partial t}(0, \vec{r})=-\frac{1}{\hbar} \mathcal{E}_{0}, \frac{\partial \beta}{\partial t}(T, \vec{r})=-\frac{1}{\hbar} \mathcal{E}_{T}
$$

Finally, the vector potential and the scalar potential in the chosen gauge are then given by

$$
\begin{aligned}
\vec{A}(t, \vec{r}) & =\vec{\chi}(t, \vec{r})+\frac{\hbar}{q} \nabla \beta(t, \vec{r}), \\
\phi(t, \vec{r}) & =\Phi(t, \vec{r})-\frac{\hbar}{q} \frac{\partial \beta}{\partial t},
\end{aligned}
$$

and the electric and magnetic fields are given by Eq. (7).

The above boundary conditions guarantee that the magnetic field is continuous at the initial and final time. To make the electric field continuous at the initial and final time, we also impose

$$
\frac{\partial \vec{\chi}}{\partial t}(0, \vec{r})=0, \frac{\partial \vec{\chi}}{\partial t}(T, \vec{r})=0 .
$$

\section{ENERGY EIGENSTATES OF A PENNING TRAP}

Let us introduce cylindrical coordinates $\{r, \theta, z\}$ where $x=r \cos \theta$ and $y=r \sin \theta$, and define the orthogonal unit vectors

$$
\hat{r}=\left(\begin{array}{c}
\cos \theta \\
\sin \theta \\
0
\end{array}\right), \hat{\theta}=\left(\begin{array}{c}
-\sin \theta \\
\cos \theta \\
0
\end{array}\right), \hat{z}=\left(\begin{array}{l}
0 \\
0 \\
1
\end{array}\right) .
$$

For the Penning trap, we assume a homogeneous magnetic field in $z$ direction, $\vec{B}=B_{z} \hat{z}$, and an electrostatic field of the form $\vec{E}=E_{r} \hat{r}+E_{\theta} \hat{\theta}+E_{z} \hat{z}$, where

$$
E_{r}=\frac{m \omega_{z}^{2}}{2 q} r, E_{\theta}=0, E_{z}=-\frac{m \omega_{z}^{2}}{q} z
$$

The vector potential and the scalar potential can be written as

$$
\vec{A}=\frac{r B_{z}}{2} \hat{\theta}, \phi=\frac{m \omega_{z}^{2}}{4 q}\left(2 z^{2}-r^{2}\right) .
$$

The corresponding Hamiltonian reads

$$
H=-\frac{\hbar^{2}}{2 m} \Delta+\frac{m}{2} \widetilde{\omega}^{2} r^{2}-\omega L_{z}+\frac{1}{2} m \omega_{z}^{2} z^{2},
$$

where $\widetilde{\omega}^{2}=\omega^{2}-\omega_{z}^{2} / 2, \omega=q B_{z} /(2 m)$ and $L_{z}=\frac{\hbar}{i} \frac{\partial}{\partial \theta}$ is the $z$-component of the angular momentum operator.
$L_{z}$ commutes with the rest of the Hamiltonian, so it represents a conserved quantity. The Hamiltonian (24) is separable into a Hamiltonian depending on $r$ and $\theta$ and a Hamiltonian depending solely on $z$. The corresponding Schrödinger equation can be solved by a product of a function of $r$ and $\theta$ and a function of $z$. The $z$-dependent function describes the dynamics of a harmonic oscillator with axial (angular) frequency $\omega_{z}$ which we assume to have a fixed value. Since we shall consider $B_{z}$ as the time dependent external parameter, the non-trivial part of interest is the function that depends on $r$ and $\theta$. Hence we focus solely on this part. We also assume $q B_{z}>0$. The energy eigenfunctions are

$$
\psi_{N, M, l}(r, \theta)=f_{N, M, l}(r) \exp (i M \theta),
$$

where

$$
\begin{aligned}
f_{N, M, l}(r)= & \frac{1}{\sqrt{2 \pi}} \sqrt{\frac{N !}{(N+|M|) !}} \frac{1}{l}\left[\frac{r}{\sqrt{2} l}\right]^{|M|} \\
& \times \exp \left(-\frac{r^{2}}{4 l^{2}}\right) L_{N}^{|M|}\left(\frac{r^{2}}{2 l^{2}}\right)
\end{aligned}
$$

$N, M \in \mathbb{Z}, L_{N}^{|M|}(q)$ are the generalized Laguerre Polynomials, defined by

$$
L_{N}^{a}(Q)=\frac{Q^{-a} e^{Q}}{N !} \frac{d^{N}}{d Q^{N}}\left(e^{-Q} Q^{N+a}\right),
$$

and the constant $l$ is defined by

$$
l=\sqrt{\frac{\hbar}{2 m \widetilde{\omega}}} .
$$

$M$ is the quantum number associated with the $z$ component of the angular momentum operator (i.e. $\left.L_{z} \psi_{N, M, l}(r, \theta)=M \hbar \psi_{N, M, l}(r, \theta)\right)$ and $N$ is a quantum number that determines the radial structure. $l \in \mathbb{R}$ is the characteristic radial length scale of the wavefunction; it is determined by the magnetic field $B_{z}$ and the axial frequency $\omega_{z}$ via Eq. (28). The energy eigenvalues are

$$
\mathcal{E}_{N, M}=\hbar \widetilde{\omega}(2 N+|M|+1)-\hbar \omega M,
$$

where $N=0,1, \ldots$ and $M$ is an integer. Alternatively, using $\widetilde{N}:=2 N+|M|$, the energy eigenvalues are often written as $\mathcal{E}=\hbar \widetilde{\omega}(\widetilde{N}+1)-\hbar \omega M$ with $\widetilde{N}=0,1, \ldots$. and $M=-\widetilde{N},-\widetilde{N}+2, \ldots, \widetilde{N}-2, \widetilde{N}$. For example, these eigenfunctions were previously found for $\widetilde{\omega}=\omega$ in $[24]$.

\section{VARYING THE MAGNETIC FIELD STRENGTH}

We would like to design the time dependence of the magnetic field so that the system starts from the eigenstate $\psi_{N, M, l_{0}}(r, \theta)$ at initial time $t=0$ with magnetic field $B_{z}(0)=B_{z, 0}$ and ends in the 
eigenstate $\Psi_{N, M, l_{T}}(r, \theta)$ at final time $t=T$ with magnetic field $B_{z}(T)=B_{z, T}$, i.e. $\Psi(0, r, \theta)=$ $\psi_{N, M, l_{0}}(r, \theta), \Psi(T, r, \theta)=\psi_{N, M, l_{T}}(r, \theta)$, where $l_{0}=$ $\sqrt{\hbar /(2 m \widetilde{\omega}(0))}, \quad l_{T}=\sqrt{\hbar /(2 m \widetilde{\omega}(T))}$, and $\widetilde{\omega}(t)=$ $\sqrt{\left(q B_{z}(t) /(2 m)\right)^{2}-\omega_{z}^{2} / 2}$. The frequency $\omega_{z}$ should be kept constant. Of course, this can be done in an adiabatic way but here we want to derive a shortcut to adiabaticity. As an example, we will examine a way to decrease the characteristic length scale, $l_{0} \rightarrow l_{T}$.

\section{A. Streamlined formalism}

Following the algorithm presented in Section II, we start by choosing the following ansatz for the time evolution of the wavefunction

$$
\begin{aligned}
\alpha(t, r)= & \sqrt{\frac{N !}{(N+|M|) !}} \frac{1}{\sqrt{2 \pi} l(t)}\left(\frac{r}{\sqrt{2} l(t)}\right) \\
& \times \exp \left(-\frac{r^{2}}{4 l(t)^{2}}\right) L_{N}^{|M|}\left(\frac{r^{2}}{2 l(t)^{2}}\right)
\end{aligned}
$$

and $\beta(t, \theta)=M \theta+\zeta(t)$. For the boundary conditions (13), it follows $\alpha(0, r)=f_{N, M, l_{0}}(r)$ and $\alpha(T, r)=$ $f_{N, M, l_{T}}(r)$ and so we get the condition $l(0)=l_{0}, l(T)=$ $l_{T}$. Moreover, we get $\zeta(0)=\zeta(T)=0$.

As the next step, we have to solve the main equation (9). We assume that $\vec{\chi}$ does not depend on $\theta$, i.e. $\vec{\chi}=$ $\chi_{r}(t, r) \hat{r}+\chi_{\theta}(t, r) \hat{\theta}$. Equation (9) then becomes

$$
\frac{2 m r}{q} \frac{\partial \alpha}{\partial t}-\chi_{r}\left(a+2 r \frac{\partial \alpha}{\partial r}\right)-r \frac{\partial \chi_{r}}{\partial r} \alpha=0,
$$

and Eq. (8) becomes

$$
\Phi=-\frac{q}{2 m}\left(\chi_{r}^{2}+\chi_{\theta}^{2}\right)+\frac{\hbar^{2}}{2 m q \alpha}\left(\frac{1}{r} \frac{\partial \alpha}{\partial r}+\frac{\partial^{2} \alpha}{\partial r^{2}}\right)
$$

A solution of Eq. (9) is given by

$$
\chi_{r}(t, r)=-\frac{2 m}{q} \frac{1}{r \alpha^{2}} \int_{r}^{\infty} d s s \alpha \frac{\partial \alpha}{\partial t}(t, s) .
$$

The solution when $\alpha$ and $\vec{\chi}$ depend on $\theta$ can be found in Appendix B.

For $\alpha$ given by Eq. (30), we get from Eq. (33) that

$$
\chi_{r}(t, r)=-\frac{m}{q} \frac{r l^{\prime}(t)}{l(t)}
$$

where the prime indicates a derivative with respect to time. Note that this solution is independent of the quantum numbers $N$ and $M$.

The components of the physical fields can be written as

$$
\begin{aligned}
& B_{z}(t, r)=\frac{1}{r} \frac{\partial\left(r \chi_{\theta}\right)}{\partial r} \\
& E_{r}(t, r)=-\frac{\partial}{\partial r} \Phi-\frac{\partial \chi_{r}}{\partial t}, E_{\theta}(t, r)=-\frac{\partial \chi_{\theta}}{\partial t} .
\end{aligned}
$$

We want a uniform magnetic field and a constant radial electric field $\left(E_{r}=\frac{m \omega_{z}^{2}}{2 q} r\right)$ during the whole process. To achieve a uniform magnetic field $B_{z}=B_{z}(t)$ we set

$$
\chi_{\theta}(t, r)=\frac{r}{2} B_{z}(t)+\frac{g(t)}{r},
$$

with an arbitrary function $g$. The electric field component $E_{r}$ is now

$$
\begin{aligned}
E_{r}= & \frac{M^{2} \hbar^{2}-q^{2} g(t)^{2}}{m q r^{3}} \\
& +\frac{r}{4 m q}\left(q^{2} B_{z}^{2}-\frac{\hbar^{2}}{l(t)^{4}}+\frac{4 m^{2} l^{\prime \prime}(t)}{l(t)}\right) .
\end{aligned}
$$

The demand $E_{r}=\frac{m \omega_{z}^{2}}{2 q} r$ leads to the choice $g(t)=$ $-M \hbar / q$ and

$$
B_{z}(t)=\frac{\sqrt{\hbar^{2}-4 m^{2} l(t)^{3} l^{\prime \prime}(t)+2 m^{2} \omega_{z}^{2} l(t)^{4}}}{q l(t)^{2}},
$$

where $q B_{z}(t)>0$ is assumed. The electric field components are finally

$$
E_{r}=\frac{m \omega_{z}^{2}}{2 q} r, E_{\theta}=-\frac{r}{2} B_{z}^{\prime}(t)
$$

Now, we have

$$
\chi(t, r)=\left[\frac{r}{2} B_{z}(t)-\frac{M \hbar}{q r}\right] \hat{\theta}-\frac{m}{q} \frac{r l^{\prime}(t)}{l(t)} \hat{r} .
$$

Following from Eq. (14), we get the boundary conditions for $\vec{\chi}$

$$
\begin{aligned}
\vec{\chi}(0, r) & =\vec{A}_{0}-\frac{\hbar}{q} \nabla \beta_{0}=\left(\frac{r B_{0}}{2}-\frac{\hbar M}{q r}\right) \hat{\theta}, \\
\vec{\chi}(T, r) & =\vec{A}_{T}-\frac{\hbar}{q} \nabla \beta_{T}=\left(\frac{r B_{T}}{2}-\frac{\hbar M}{q r}\right) \hat{\theta} .
\end{aligned}
$$

With the $\chi$ given in Eq. (41), this is fulfilled if $l^{\prime}(0)=0$, $l^{\prime}(T)=0, B_{z}(0)=B_{0}$, and $B_{z}(T)=B_{T}$. To fulfill the last two conditions, we have to demand $l^{\prime \prime}(0)=0$, $l^{\prime \prime}(T)=0$.

The boundary conditions of $\Phi$ are fulfilled if the conditions (17) are satisfied, i.e. if

$$
\frac{\partial \zeta}{\partial t}(0)=-\frac{1}{\hbar} \mathcal{E}_{0}, \frac{\partial \zeta}{\partial t}(T)=-\frac{1}{\hbar} \mathcal{E}_{T} .
$$

A simple choice of $\zeta$ may be a polynomial of degree 3 that obeys all of the boundary conditions on $\zeta$. Note that the magnetic and the electric fields do not depend on the choice of the time dependent global phase $\zeta(t)$.

An additional boundary condition on $l(t)$ can be derived by enforcing that the electric field is continuous at $t=0$ and $t=T$, see the conditions (20). This requires 
$B_{z}^{\prime}(0)=0$ and $B_{z}^{\prime}(T)=0$. Differentiating the expression (39) for $B_{z}(t)$ with respect to time gives

$$
B_{z}^{\prime}(t)=-\frac{2\left(l^{\prime}(t)\left[\hbar^{2}-m^{2} l(t)^{3} l^{\prime \prime}(t)\right]+m^{2} l(t)^{4} l^{\prime \prime \prime}(t)\right)}{q l^{3}(t) \sqrt{\hbar^{2}-4 m^{2} l(t)^{3} l^{\prime \prime}(t)+2 m^{2} \omega_{z}^{2} l(t)^{4}}} .
$$

Noting the boundary conditions on $l$ already derived, this requires, in addition, that $l^{\prime \prime \prime}(0)=0, l^{\prime \prime \prime}(T)=0$.

In summary, the boundary conditions for $l(t)$ are

$$
\begin{gathered}
l(0)=l_{0}=\sqrt{\frac{\hbar}{2 m \widetilde{\omega}(0)}}, l^{\prime}(0)=l^{\prime \prime}(0)=l^{\prime \prime \prime}(0)=0, \\
l(T)=l_{T}=\sqrt{\frac{\hbar}{2 m \widetilde{\omega}(T)}}, l^{\prime}(T)=l^{\prime \prime}(T)=l^{\prime \prime \prime}(T)=0 .
\end{gathered}
$$

These conditions are independent of the quantum numbers $N$ and $M$.

If $l(t)$ satisfies these boundary conditions, the corresponding magnetic field and electric field are given by Eqs. (39) and (40), and fulfill $\nabla \cdot \vec{E}=0$ and $\nabla \times \vec{B}=0$. They are also independent of the quantum numbers $N$ and $M$. If the system starts in the corresponding eigenstate and if these fields are implemented, then the system will end with fidelity 1 in the final state. We will show that these schemes which do not change the quantum number could be alternatively derived using an invariant based approach.

\section{B. Invariant based approach}

A Lewis-Riesenfeld invariant is a Hermitian operator $I(t)$ fulfilling

$$
\frac{\partial}{\partial t} I(t)=\frac{i}{\hbar}[I(t), H(t)]_{-},
$$

where $H(t)$ is the Hamiltonian for the system. If we disregard again the $z$ dependent part, we find the following invariant for the Hamiltonian (24),

$$
\begin{aligned}
I(t)= & -\frac{l(t)^{2}}{\hbar^{2}} \Delta-2 m l^{\prime}(t) l(t)\left(\frac{\hbar}{i} \frac{\partial}{\partial r}\right) r \\
& +\left(m^{2} l^{\prime}(t)^{2}+\frac{\hbar^{2}}{4 l(t)^{2}}\right) r^{2}
\end{aligned}
$$

where the function $l(t)$ has to be a solution of the following Ermakov-like equation

$$
4 m^{2} \frac{l^{\prime \prime}(t)}{l(t)}+4 m^{2} \widetilde{\omega}(t)^{2}-\frac{\hbar^{2}}{l(t)^{4}}=0 .
$$

(In [22], the case $\widetilde{\omega}=\omega$ was examined and an invariant was constructed. Eigenstates of this invariant which are simultaneously eigenstates of $L_{z}$ were also constructed indirectly.) An explicit expression of the eigenstates of $I$ is

$$
\begin{aligned}
& \Gamma_{N, M}(t, r, \theta)=\frac{1}{\sqrt{2 \pi}} \sqrt{\frac{N !}{(N+|M|) !}} \frac{1}{l(t)}\left[\frac{r}{\sqrt{2} l(t)}\right]^{|M|} \\
& \quad \times \exp \left(-\frac{r^{2}}{4 l(t)^{2}}\right) L_{N}^{|M|}\left(\frac{r^{2}}{2 l(t)^{2}}\right) \exp (i M \theta) \\
& \quad \times \exp \left(\frac{i m l^{\prime}(t)}{2 \hbar l} r^{2}\right),
\end{aligned}
$$

where $N, M \in \mathbb{Z}$ and $l(t)$ is a solution of Eq. (48). The corresponding eigenvalue of $I$ is $(2 N+|M|+1) \hbar^{2}$ and the corresponding eigenvalue of $L_{z}$ is $M \hbar$. A more general invariant (which allows for a time dependent mass) was described in [25]. An eigenstate of a Lewis-Riesenfeld invariant is a solution to the Schrödinger equation for $H(t)$ up to a time dependent phase $\Pi_{N, M}(t)$ [22], which is here given by

$$
\Pi_{N, M}(t)=\int_{0}^{t} d t^{\prime}\left(M \omega\left(t^{\prime}\right)-\frac{(N+1) \hbar}{2 m l\left(t^{\prime}\right)^{2}}\right) .
$$

The idea is to do inverse-engineering by demanding that the system follows the state $\Psi(t, r, \theta)=$ $\Gamma_{N, M}(t, r, \theta) e^{i \Pi_{N, M}(t)}$. First, we choose an auxiliary function $l(t)$ (which has to fulfill different conditions at initial and final time, see below) and then we get $\widetilde{\omega}(t)$ from Eq. (48). At initial and final time the eigenstates of the invariant should coincide with the eigenstates of the Hamiltonian, i.e. $[I(0), H(0)]_{-}=0=[I(T), H(T)]_{-}$. Therefore, we have to impose the following boundary conditions for the auxiliary function $l(t)$ :

$$
\begin{array}{lll}
l(0)=l_{0}=\sqrt{\frac{\hbar}{2 m \widetilde{\omega}(0)}}, & l(T)=l_{T}=\sqrt{\frac{\hbar}{2 m \widetilde{\omega}(T)},} \\
l^{\prime}(0)=0, & l^{\prime}(T)=0 .
\end{array}
$$

From these boundary conditions and Eq. (48), it also follows that $l^{\prime \prime}(0)=0$ and $l^{\prime \prime}(T)=0$.

An additional boundary condition on $l(t)$ can be derived by enforcing that the electric field is continuous at $t=0$ and $t=T$. This leads to $B_{z}^{\prime}(0)=B_{z}^{\prime}(T)=0$ or $l^{\prime \prime \prime}(0)=l^{\prime \prime \prime}(T)=0$. The complete list of boundary conditions is equivalent to Eq. (45) above. As already mentioned, we can now design first an auxiliary function $l(t)$ fulfilling the above boundary conditions and then calculate $\widetilde{\omega}(t)$ and hence the magnetic field strength $B_{z}(t)$ from Eq. (48). The resulting formula for $B_{z}(t)$ is the same as Eq. (39) above.

Summarizing, the streamlined fast-forward formalism and the invariant based approach provide two ways to find the same boundary conditions for the auxiliary function $l(t)$ in this setting. So, for varying the magnetic field strength, both formalisms are equivalent as they both require that one chooses the auxiliary function $l(t)$ fulfilling these boundary conditions and then the corresponding physical potentials can be calculated in the same way. In the following we look at a numerical example of this procedure. 


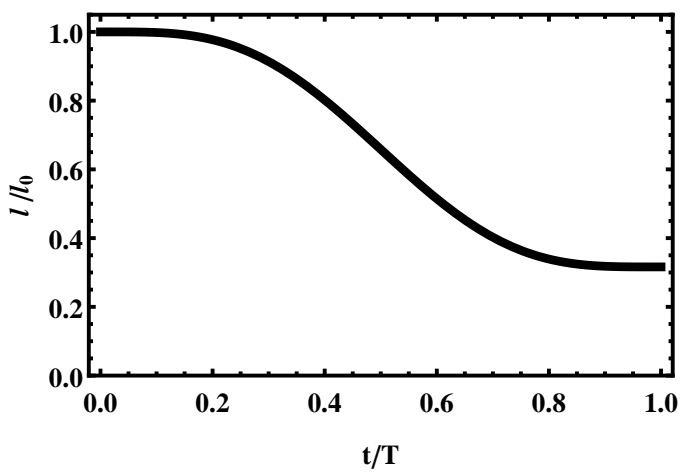

FIG. 1: Auxiliary function $l(t)$ versus $t$.

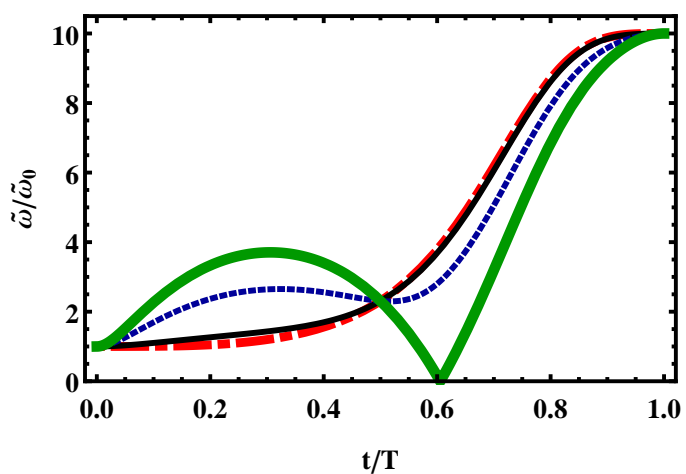

FIG. 2: (Color online) Frequency ratio $\widetilde{\omega}(t) / \widetilde{\omega}(0)$ versus $t$; $\mu \rightarrow \infty$ (red, dashed-dotted line), $\mu=3$ (black, solid line), $\mu=1$ (blue, dotted line), $\mu=0.672$ (green, thick, solid line).

\section{Numerical Example}

Let us first set $l(t)=l_{0} \lambda(\tau)$ where $\tau=t / T$. From the above formalism, the time dependence of $\widetilde{\omega}$ follows as

$$
\widetilde{\omega}(t)=\widetilde{\omega}(0) \frac{1}{\lambda(\tau)^{2}} \sqrt{1-\frac{\lambda(\tau)^{3} \lambda^{\prime \prime}(\tau)}{\mu^{2}}},
$$

where $\mu=T \widetilde{\omega}(0) . \quad \mu$ can be seen as the final time in units of $1 / \widetilde{\omega}(0)$. Therefore, decreasing $\mu$ corresponds to decreasing the total time $T$ of the process, with fixed $\widetilde{\omega}(0)$ (i.e. fixed $\omega_{0}=\frac{q B_{z}(0)}{2 m}>0$ and fixed $\omega_{z}$ ). The limit $\mu \rightarrow \infty$ would correspond to the adiabatic limit where we get $\widetilde{\omega}(t) / \widetilde{\omega}(0) \rightarrow \frac{1}{\lambda^{2}(\tau)}$.

The corresponding magnetic field would then be given by Eq. (39) or in dimensionless variables

$$
B_{z}(t)=\frac{\hbar}{q l_{0}^{2}} \frac{1}{\lambda(\tau)^{2}}\left[1-\frac{\lambda(\tau)^{3} \lambda^{\prime \prime}(\tau)}{\mu^{2}}+\frac{\nu^{2}}{2-\nu^{2}} \lambda(\tau)^{4}\right]^{1 / 2}
$$

where $\nu=\frac{\omega_{z}}{\omega_{0}}$ is the ratio between the two initial frequencies. This parameter $\nu$ is independent of the total time $T$. We want to have a trap setting at initial and final time, i.e. $\widetilde{\omega}(0)^{2}$ and $\widetilde{\omega}(T)^{2}$ should be positive. From

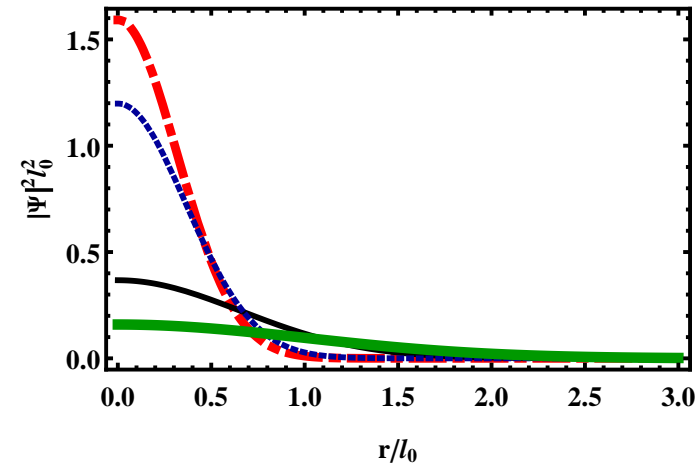

FIG. 3: (Color online) Time evolution of $|\Psi(t, r)|^{2}$ with $N=$ $M=0 ; t=0$ (green, thick, solid line), $t=T / 2$ (black, thin, solid line), $t=3 T / 4$ (blue, dotted line), $t=T$ (red, dasheddotted line).

this, $\nu$ must be in the range $0 \leq \nu<\sqrt{2} \min \left\{1, \omega_{T} / \omega_{0}\right\}$ where $\omega_{T}=\frac{q B_{z}(T)}{2 m}>0$.

Assuming a polynomial form of $\lambda(\tau)$ and using the above conditions, $\lambda(\tau)$ can be expressed as

$$
\begin{aligned}
\lambda(\tau)= & 1-20\left(l_{T} / l_{0}-1\right) \tau^{7}+70\left(l_{T} / l_{0}-1\right) \tau^{6} \\
& -84\left(l_{T} / l_{0}-1\right) \tau^{5}+35\left(l_{T} / l_{0}-1\right) \tau^{4}
\end{aligned}
$$

The final value of the magnetic field is chosen in this example such that $\widetilde{\omega}(T) / \widetilde{\omega}(0)=c=10$ (i.e. $l_{T}=l_{0} / \sqrt{10}$ ). The ratio between initial and final magnetic field is then

$$
\frac{B_{z}(T)}{B_{z}(0)}=\sqrt{c^{2}\left(1-\frac{\nu^{2}}{2}\right)+\frac{\nu^{2}}{2}}
$$

Fig. 1 is the corresponding plot of $l(t)$.

Fig. 2 shows $\widetilde{\omega}(t)$ for different values of $\mu$. For $\mu \approx$ 0.672 (green, thick, solid line) $\widetilde{\omega}(t)^{2}>0$ is no longer fulfilled for all times. The requirement that $B_{z}(t) \in \mathbb{R}$ for all times results in a type of quantum speed limit of the form

$$
\mu \geq \max _{\tau \in[0,1]}\left[\frac{\lambda(\tau)^{3} \lambda^{\prime \prime}(\tau)}{1+\nu^{2}\left(2-\nu^{2}\right)^{-1} \lambda(\tau)^{4}}\right]^{1 / 2} .
$$

As an example, the wavefunction at different times with $N=M=0$ can be seen in Fig. 3. The shown time evolution is independent of $\mu, \nu$ and depends only on the chosen form of $l(t)$.

\section{Superposition}

The electric and magnetic fields derived in the previous subsection are independent of the quantum numbers $N$ and $M$. Therefore, the fields can be also applied to a superposition of different eigenstates with initial magnetic field $B_{0}$ and they will produce a superposition of eigenstates with final magnetic field $B_{T}$ with the same 


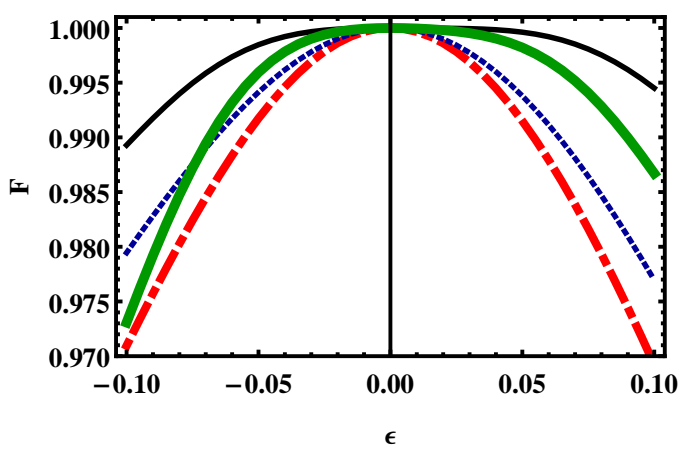

FIG. 4: (Color online) Fidelity $F$ versus error $\epsilon ; \mu=1, \nu=0.1$ (blue, dotted line); $\mu=1, \nu=1$ (red, dashed-dotted line); $\mu=3, \nu=0.1$ (black, thin, solid line); $\mu=3, \nu=1$ (green, thick, solid line).

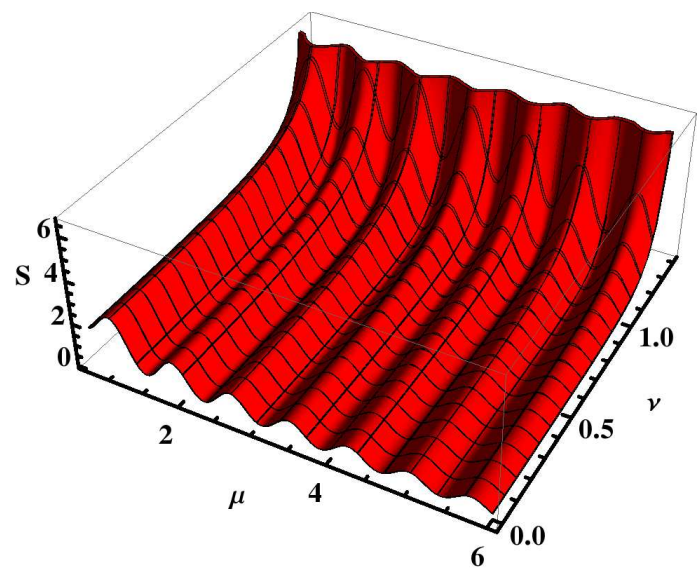

FIG. 5: (Color online) Systematic error sensitivity $S$ versus $\mu$ and $\nu$.

populations as initially. Let us assume an initial wavefunction of the form

$$
\Psi(0, r, \theta)=\sum_{N, M} c_{N, M} \Gamma_{N, M}(0, r, \theta)
$$

where $\Gamma_{N, M}(t, r, \theta)$ are the eigenfunctions of the invariant given in Eq. (49) and $c_{N, M}$ are (constant) complex coefficients. Then it follows that the state at final time will be

$$
\Psi(T, r, \theta)=\sum_{N, M} c_{N, M} e^{i \Pi_{N, M}(T)} \Gamma_{N, M}(T, r, \theta),
$$

where $\Pi_{N, M}$ is given in Eq. (150), so the populations in the different eigenstates will be the same as initially, i.e. $\left|c_{N, M} e^{i \Pi_{N, M(t)}}\right|^{2}=\left|c_{N, M}\right|^{2}$.

\section{E. Stability}

It is important that the scheme is not only fast but also stable concerning errors in the implementation. We want to examine the stability of the protocol if there is a systematic error in the magnetic field $B_{z}(t)$. We assume that the magnetic field is correctly implemented before and after the process, for $t \leq 0$ and $t \geq T$. Nevertheless, during the change of the magnetic field for for $0<t<T$, we assume that an inaccurate magnetic field $\mathcal{B}_{\epsilon}(t)=B_{z}(t)(1+\epsilon)$ is implemented, where $B_{z}(t)$ is the correct one and $\epsilon$ a small relative systematic error which is unknown but constant.

We will examine the final fidelity as a function of $\epsilon$ for $N=M=0$. The initial state is still $\Psi_{\epsilon}(0)=$ $\psi_{N=0, M=0, l_{0}}$. The solution of the Schrödinger equation is then still given by $\Psi_{\epsilon}(t)=\Gamma_{0,0}(t) e^{i \Pi_{0,0}(t)}$ (see Eq. (49)) with $l(t)$ replaced by $\ell_{\epsilon}(t)$, a solution of

$$
4 m^{2} \frac{\ell_{\epsilon}^{\prime \prime}(t)}{\ell_{\epsilon}(t)}+4 m^{2}\left[\left(\frac{q \mathcal{B}_{\epsilon}(t)}{2 m}\right)^{2}-\frac{\omega_{z}^{2}}{2}\right]-\frac{\hbar^{2}}{\ell_{\epsilon}(t)^{4}}=0
$$

with $\ell_{\epsilon}(0)=l_{0}$ and $\ell_{\epsilon}^{\prime}(0)=0$. The fidelity at $t=T$ is now

$$
\begin{aligned}
F & =\left|\left\langle\Psi(T) \mid \Psi_{\epsilon}(T)\right\rangle\right| \\
& =\frac{2 l(T) \ell_{\epsilon}(T)}{\sqrt{\left(l(T)^{2}+\ell_{\epsilon}(T)^{2}\right)^{2}+\frac{4 m}{\hbar^{2}} l(T)^{4} \ell_{\epsilon}(T)^{2} \ell_{\epsilon}^{\prime}(T)^{2}}}(.59)
\end{aligned}
$$

Let $l(t)$ be given again as in Eq. (54). We once again fix $\widetilde{\omega}(T) / \widetilde{\omega}(0)=c=10$, noting that the magnetic field is assumed to be error-free at the initial and final time. With these values fixed, the fidelity $F$ only depends on $\mu$, $\nu$ and $\epsilon$. Note $\nu$ must be in the range $0 \leq \nu<\sqrt{2}$. The fidelity $F$ for different combinations of $\mu$ and $\nu$ versus $\epsilon$ is shown in Fig. 4. One still gets a high fidelity even if there is a small, systematic error in the implementation of the magnetic field during the scheme. The scheme is, in some range, stable concerning this type of systematic error.

A sensitivity $S$ of the scheme versus this systematic error can be defined as the negative curvature of the fidelity at $\epsilon=0$, i.e. $S=-\left.\frac{\partial^{2} F}{\partial \epsilon^{2}}\right|_{\epsilon=0}$. This sensitivity $S$ versus $\mu$ and $\nu$ is shown in Fig. [5] The sensitivity is increasing with increasing ratio $\nu$ for fixed $\mu$. For fixed $\nu$ the sensitivity shows an oscillating behavior with increasing $\mu$. The sensitivity for arbitrary $N$ and $M$ is treated in Appendix C.

In order to have even more stability against systematic error in the magnetic field one could design a different $l(t)$ which minimizes the sensitivity $S$ and still fulfills the necessary boundary conditions (a similar strategy could also be applied to other types of systematic errors or random errors). As shown in Appendix [C] it would be sufficient to minimize $S$ only for $N, M=0$.

\section{DISCUSSION}

We have put forward shortcuts to adiabaticity for a charged particle in an electromagnetic field focusing on a 
change of the radial spread in a Penning trap by modifying the magnetic field intensity. Two methods have been used for this: a streamlined version of the fast-forward formalism for an electromagnetic field, and an invariant based procedure. We have shown their equivalence for this operation. In general the fast-forward formalism presented in this paper could be applied to other tasks for which the invariant approach is not well suited, such as transformations for individual states [19]. We also found that a type of quantum speed limit applies. Moreover, we have examined the scheme in the case of a systematic error in the magnetic field and shown its stability.

\section{Acknowledgments}

We are grateful to David Rea for useful discussion and commenting on the manuscript. This work was supported by the Basque Country Government (Grant No. IT472-10), Ministerio de Economía y Competitividad (Grant No. FIS2012-36673-C03-01), and the program UFI 11/55 of the Basque Country University.

\section{Appendix A: Boundary conditions for $\Phi(t, \vec{r})$}

Because $\psi_{0}(\vec{r}) \equiv \alpha_{0}(\vec{r}) e^{i \beta_{0}(\vec{r})}$ should be an energy eigenvector of the Hamiltonian $H_{0}$ with eigenvalue $\mathcal{E}_{0}$, it follows from the real part of the corresponding stationary Schrödinger equation that

$0=-\frac{\hbar^{2}}{2 m} \Delta \alpha_{0}+\frac{1}{2 m}\left(q \vec{A}_{0}-\hbar \nabla \beta_{0}\right)^{2} \alpha_{0}+\left(q \phi-\mathcal{E}_{0}\right) \alpha_{0}$,

and from the imaginary part that

$0=\frac{\hbar}{2 m} \nabla\left(q \vec{A}_{0}-\hbar \nabla \beta_{0}\right) \alpha_{0}+\frac{\hbar}{m}\left(q \vec{A}_{0}-\hbar \nabla \beta_{0}\right) \nabla \alpha_{0}$.

Eq. (9) at initial time becomes

$$
\frac{1}{q} \frac{\partial \alpha}{\partial t}(0, \vec{r})=\frac{1}{2 m}\left(\nabla \vec{\chi}_{0}\right) \alpha_{0}+\frac{1}{m} \vec{\chi}_{0} \nabla \alpha_{0}=0,
$$

because of Eq. (A2), and $\vec{\chi}_{0}=\vec{A}_{0}-\frac{\hbar}{q} \nabla \beta_{0}$.

Eq. (8) at initial time becomes

$$
\Phi(0, \vec{r})=\frac{\hbar^{2}}{2 m q \alpha_{0}} \Delta \alpha_{0}-\frac{q}{2 m} \vec{\chi}_{0}^{2}=\phi_{0}-\frac{\mathcal{E}_{0}}{q}
$$

because of Eq. (A1). Similar calculations also apply to the final time.

\section{Appendix B: Solution of the main equations in polar coordinates}

We assume that $\alpha(t, r, \theta)$ is given. We set

$$
\vec{\chi}=\chi_{r}(t, r, \theta) \hat{r}+\chi_{\theta}(t, r, \theta) \hat{\theta} .
$$

The main equation (9) now becomes

$$
\begin{array}{r}
\frac{2 m r}{q} \frac{\partial \alpha}{\partial t}-2 \chi_{\theta} \frac{\partial \alpha}{\partial \theta}-\frac{\partial \chi_{\theta}}{\partial \theta} \alpha-\chi_{r}\left(\alpha+2 r \frac{\partial \alpha}{\partial r}\right) \\
-r \frac{\partial \chi_{r}}{\partial r} \alpha=0
\end{array}
$$

and Eq. (8) becomes

$$
\begin{aligned}
\Phi= & -\frac{q}{2 m}\left(\chi_{r}^{2}+\chi_{\theta}^{2}\right) \\
& +\frac{\hbar^{2}}{2 m q \alpha}\left(\frac{1}{r^{2}} \frac{\partial^{2} \alpha}{\partial \theta^{2}}+\frac{1}{r} \frac{\partial \alpha}{\partial r}+\frac{\partial^{2} \alpha}{\partial r^{2}}\right) .
\end{aligned}
$$

A solution of the main equation (B2) for $\chi_{r}$ in terms of $w=\alpha^{2}$ and $\chi_{\theta}$ can be written down,

$$
\begin{aligned}
\chi_{r} & =-\frac{1}{r w} \int_{r}^{\infty} d s\left[\frac{m s}{q} \frac{\partial w}{\partial t}(t, s, \theta)\right. \\
& \left.-\chi_{\theta}(t, s, \theta) \frac{\partial w}{\partial \theta}(t, s, \theta)-w(t, s, \theta) \frac{\partial \chi_{\theta}}{\partial \theta}(t, s, \theta)\right] .
\end{aligned}
$$

Thus a function $\chi_{\theta}$ can be chosen to determine (together with the chosen $\alpha$ ) the function $\chi_{r}$.

Alternatively, a solution of the main equation for $\chi_{\theta}$ in terms of $a$ and $\chi_{r}$ is given by

$$
\begin{aligned}
\chi_{\theta} & =-\frac{f_{\theta}(r, t)}{a}+\frac{1}{a} \int_{0}^{\theta} d \rho\left\{\frac{m r}{q} \frac{\partial a^{2}}{\partial t}(r, \rho, t)\right. \\
& -r \chi_{r}(r, \rho, t) \frac{\partial a^{2}}{\partial r}(r, \rho, t) \\
& \left.-a^{2}(r, \rho, t)\left[\chi_{r}(r, \rho, t)+r \frac{\partial \chi_{r}}{\partial r}(r, \rho, t)\right]\right\},
\end{aligned}
$$

where $\chi_{\theta}(r, \theta+2 \pi, t)=\chi_{\theta}(r, \theta, t)$.

\section{Appendix C: Fidelity and sensitivity for arbitrary $N$ and $M$}

For arbitrary $N$ and $M$ we get for the fidelity $F_{N, M}$, by using [26],

$$
\begin{aligned}
F_{N, M} & =\left|\left\langle\Psi(T) \mid \Psi_{\epsilon}(T)\right\rangle\right| \\
& =Q^{1+|M|}\left|P_{N}^{(|M|, 0)}\left(1-2 Q^{2}\right)\right|,
\end{aligned}
$$

where $P$ are Jacobi's polynomials and

$$
Q=\frac{2 l(T) \ell_{\epsilon}(T)}{\sqrt{\left(l(T)^{2}+\ell_{\epsilon}(T)^{2}\right)^{2}+\frac{4 m}{\hbar^{2}} l(T)^{4} \ell_{\epsilon}(T)^{2} \ell_{\epsilon}^{\prime}(T)^{2}}}
$$

The result is valid for an arbitrary function $l(t)$. Note that $F_{N=0, M=0}=Q=F$, this is the special case given in Eq. (59). 
The general sensitivity $S_{N, M}=-\left.\frac{\partial^{2} F_{N, M}}{\partial \epsilon^{2}}\right|_{\epsilon=0}$ is

$$
S_{N, M}=\left.\frac{\partial F_{N, M}}{\partial Q}\right|_{Q=1} \times S_{N=0, M=0}
$$

where $S_{N=0, M=0}=S$ is the special case discussed in the main text. The factor $\left.\frac{\partial F_{N, M}}{\partial Q}\right|_{Q=1}$ only depends on $N$ and $M$ and is independent of the chosen $l(t)$.

$043436(2012)$

[1] E. Torrontegui, S. Ibáñez, S. Martínez-Garaot, M. Modugno, A. del Campo, D. Guéry-Odelin, A. Ruschhaupt, X. Chen, and J.G. Muga, Adv. At. Mol. Opt. Phys. 62, 117 (2013).

[2] X. Chen, A. Ruschhaupt, S. Schmidt, A. del Campo, D. Guéry-Odelin, and J. G. Muga, Phys. Rev. Lett. 104, 063002 (2010).

[3] J. F. Schaff, X. L. Song, P. Vignolo, and G. Labeyrie, Phys. Rev. A 82033430 (2010); Phys. Rev. A 83, 059911(E) (2011).

[4] J. F. Schaff, X. L. Song, P. Capuzzi, P. Vignolo, and G. Labeyrie, Europhys. Lett. 93, 23001 (2011).

[5] M. Palmero, S. Martínez-Garaot, J. Alonso, J. P. Home, and J. G. Muga, in preparation.

[6] J. G. Muga, X. Chen, A. Ruschhaupt, and D. GuéryOdelin, J. Phys. B: At. Mol. Opt. Phys. 42, 241001 (2009).

[7] D. Guéry-Odelin, J. G. Muga, M. J. Ruiz-Montero, and E. Trizac, Phys. Rev. Lett. 112, 180602 (2014).

[8] Y. Li, L. A. Wu, and Z. D. Wang, Phys. Rev. A 83, 043804 (2011).

[9] J.-Q. Zhang, L. Yong, and M. Feng, J. Phys.: Condens. Matter 25, 142201 (2013).

[10] P. Salamon, K. H. Hoffmann, Y. Rezek, and R. Kosloff, Phys. Chem. Chem. Phys. 11, 1027 (2009).

[11] K. H. Hoffmann, P. Salamon, Y. Rezek, and R. Kosloff, EPL 96, 60015 (2011).

[12] S. Choi, R. Onofrio, and B. Sundaram, Phys. Rev. A 84, 051601(R) (2011).

[13] S. Choi, R. Onofrio, and B. Sundaram, Phys. Rev. A 86,
[14] X. Chen and J. G. Muga, Phys. Rev. A 82, 053403 (2010).

[15] E. Torrontegui, X. Chen, M. Modugno, A. Ruschhaupt, D. Guéry-Odelin, and J. G. Muga, Phys. Rev. A 85, 033605 (2012).

[16] K Blaum, Yu. N. Novikov, and G. Werth, Contemporary Physics 51, 149 (2010).

[17] S. Masuda and K. Nakamura, Phys. Rev. A 78, 062108 (2008).

[18] S. Masuda and K. Nakamura, Proc. R. Soc. A 466, 1135 (2010).

[19] E. Torrontegui, S. Martínez-Garaot, A. Ruschhaupt, and J. G. Muga, Phys. Rev. A 86, 013601 (2012).

[20] E. Torrontegui, S. Martínez-Garaot, M. Modugno, Xi Chen, and J. G. Muga, Phys. Rev. A 87, 033630 (2013).

[21] S. Masuda, K. Nakamura, and A. del Campo, Phys. Rev. Lett. 113, 063003 (2014).

[22] H.R. Lewis and W.B. Riesenfeld, J. Math. Phys. 10, 1458 (1969).

[23] S. Masuda and K. Nakamura, Phys. Rev. A 84, 043434 (2011).

[24] P. E. de Brito and H. N. Nazareno, Eur. J. Phys. 28, 9 (2007).

[25] G. Fiore and L. Gouba, J. Math. Phys. 52, 103509 (2011).

[26] I. S. Gradshteyn and I. M. Ryzhik, Table of Integrals, Series, and Products (Elsevier, Amsterdam, 2007), Seventh Edition, section 7.414, p. 810. 